\title{
LONG PERIOD VARIABLE STARS IN THE HIPPARCOS OBSERVING PROGRAM
}

\author{
D. BARTHES ${ }^{2}$, H. BOUGHALEB ${ }^{1}$, F. FIGUERAS ${ }^{2}$, E. G. FOSTER ${ }^{3}$, \\ J. A. MATTEI ${ }^{3}$, M. O. MENNESSIER ${ }^{1}$, E. O. WAAGEN ${ }^{3}$ \\ ${ }^{1}$ CNRS, Groupe de Recherche en Astronomie et Astrophysique de Languedoc/Universite \\ Montpellier II, F-34095 Montpellier, cedex 5, France \\ ${ }^{2}$ Universitat de Barcelona, Dept. d'Astronomia i Meteorologia, Avgda. Diagonal. 647, \\ E-08028 Barcelona, Spain \\ ${ }^{3}$ AAVSO, 25 Birch Street, Cambridge, Massachusetts 02138, USA
}

\begin{abstract}
Among the long period variable stars included in the Hipparcos observing program, 245 large-amplitude ones require brightness predictions during the mission in order to allocate the necessary observing time. We present the computation of the light ephemerides with numeric and symbolic methods using AAVSO's 75-year data on maxima and minima dates and magnitudes and 20 years of individual observations; the evaluation and revision of the ephemerides using ground-based observations compiled monthly by the AAVSO from observers world-wide and real-time monitoring of ephemerides stars from satellite observations performed at ESOC; the statistical results derived from one-and-a-half years of simultaneous ground-based and Hipparcos observations. We show the usefulness of all this work in understanding the physics of these pulsating variable stars.
\end{abstract}

Among the long period variable stars (LPVs) included in the Hipparcos observing program, 245 large amplitude ones require brightness predictions in order to allocate the necessary observing time. These stars do not have strictly constant amplitudes or periods, so long-term data and specific methods are needed for predicting their brightness and up-to-date observations are necessary for confirming and refining these predictions.

The computation of the light ephemerides with symbolic (rules) and numeric (Fourier) methods have been carried out at CNRS/Universite Montpellier II using AAVSO's 75-year data on maxima and minima dates and magnitudes and 20 years of individual observations. The rules and the Fourier methods used act in validating each other. Furthermore, the long-term rules allow us to suspect the presence of some Fourier components that could not be detected from numerical analysis over only 20 years of data.

The evaluation and revision of the ephemerides are done using ground-based observations ( 6000 per month) contributed by 600 amateur astronomers around the world. This activity is coordinated, observations compiled and digitized monthly, and the ephemerides compared with recent observations by the AAVSO. When discrepancies are found, the ephemerides are revised at CNRS/Universite Montpellier II, using various methods such as shifting, dilating, or contracting the predicted light curve, selecting another set of frequencies, and refitting them on the past and/or recent data.

Statistical studies comparing the ground-based observations and predicted magnitudes over two years showed that there was no significant systematic error in the predictions. Comparison between predicted and observed ground-based $(\mathrm{V})$ and Hipparcos $(\mathrm{H})$ magnitudes showed systematic difference in that the mean value of $\Delta H$ minus $\Delta \mathrm{V}$ increased with later spectral type. This result pointed out that the $H \cdot V$ relationship was in need of revision.

The pertinent power spectra and the set of rules obtained for the prediction of the light curves have astrophysical applications in that they are useful tools in determining the pulsation modes of the Hipparcos LPVs, the evolution of the amplitudes, and the phases, and therefore in validating the theoretical models.

We thank the variable star observers worldwide for their ground-based observations and we gratefully acknowledge the financial support provided by the Regional Council of LanguedocRoussillon for computing, the CICYT under contract ESP88-0731 and the Hispano-French Integrated Actions Program, and NASA under grant NAGW 1493 to the AAVSO for providing the data support. 